\title{
Personalizing Persuasive Strategies in Gameful Systems to Gamification User Types
}

\author{
Rita Orji ${ }^{1}$, Gustavo F. Tondello ${ }^{2,3}$, Lennart E. Nacke ${ }^{2,3,4}$ \\ ${ }^{1}$ Faculty of Computer Science, Dalhousie University, Halifax, NS, Canada \\ ${ }^{2}$ HCI Games Group, Games Institute, ${ }^{3}$ Cheriton School of Computer Science, ${ }^{4}$ Drama and Speech \\ Communication and Stratford Campus, University of Waterloo, ON, Canada \\ rita.orji@dal.ca,gustavo@tondello.com, lennart.nacke@acm.org
}

\begin{abstract}
Persuasive gameful systems are effective tools for motivating behaviour change. Research has shown that tailoring these systems to individuals can increase their efficacy; however, there is little knowledge on how to personalize them. We conducted a large-scale study of 543 participants to investigate how different gamification user types responded to ten persuasive strategies depicted in storyboards representing persuasive gameful health systems. Our results reveal that people's gamification user types play significant roles in the perceived persuasiveness of different strategies. People scoring high in the 'player' user type tend to be motivated by competition, comparison, cooperation, and reward while 'disruptors' are likely to be demotivated by punishment, goal-setting, simulation, and self-monitoring. 'Socialisers' could be motivated using any of the strategies; they are the most responsive to persuasion overall. Finally, we contribute to $\mathrm{CHI}$ research and practice by offering design guidelines for tailoring persuasive gameful systems to each gamification user type.
\end{abstract}

\section{Author Keywords}

Persuasive strategies; personalization; gamification; gameful design; Hexad; behaviour change; risky health behavior.

\section{ACM Classification Keywords}

H.5.m. Information interfaces and presentation (e.g., HCI): Miscellaneous; H.1.2. User/Machine Systems: Human factors; K.8.0. Personal Computing: Games.

\section{INTRODUCTION}

Gamification, the use of game design elements in non-game contexts [16], is a way to design engaging systems for motivating behaviour change, influencing people to adopt healthy habits, and promoting learning [33,40,60,70]. Many gameful systems use persuasive strategies [22] to motivate users to adopt specific behaviours $[1,32]$. One application area that has gained attention is 'persuasive gameful health

\footnotetext{
Permission to make digital or hard copies of all or part of this work for personal or classroom use is granted without fee provided that copies are not made or distributed for profit or commercial advantage and that copies bear this notice and the full citation on the first page. Copyrights for components of this work owned by others than ACM must be honored. Abstracting with credit is permitted. To copy otherwise, or republish, to post on servers or to redistribute to lists, requires prior specific permission and/or a fee. Request permissions from permissions@acm.org.
}

CHI 2018, April 21-26, 2018, Montreal, QC, Canada

(C) Owners/Authors, 2018. This is the author's version of the work. It is posted here for your personal use. Not for redistribution. The definitive Version of Record was published in CHI '18 Proceedings of the $2018 \mathrm{CHI}$ Conference on Human Factors in Computing Systems.

DOI: https://doi.org/10.1145/3173574.3174009 applications', which attempts to help people adopt healthy behaviours and avoid risky behaviours [18,40,60]. Persuasive gameful health applications have been applied in motivating people to increase their physical activity $[11,24,31,45,72,80]$, reduce medication misuse $[2,67]$, comply with blood glucose monitoring [10], improve overall wellbeing and flourishing [30,50], reduce stress and anxiety [15], and avoid risky behaviours [36,38].

Most of these existing applications adopt the one-size-fitsall approach in their design. However, research has shown that this approach may be ineffective for persuasion because different types of users are motivated by different persuasive strategies $[42,59,61,62]$ and game design elements [75,77]. Therefore, persuasive gameful systems are more effective at promoting behaviour change if they are personalized to the user types [42,57]. Despite this growing evidence on the need to tailor persuasive gameful systems, there is still little knowledge on how to personalize and the most effective ways to personalize persuasive gameful applications to various user types [7,52]. Tondello et al. [76] suggested a tailoring approach based on adapting the activities that a user carries out in the system. Orji et al. [61] investigated how persuasive game applications can be personalized by tailoring the persuasive strategies to various personality types. However, we still lack research on how to tailor persuasive strategies, which are the fundamental building blocks of persuasive gameful applications, to an individual's gamification user type. The Hexad gamification user types is the first user typology that is specifically developed for studying user's preferences in gameful systems. It has been validated and showed test-retest reliability [77]. Furthermore, it has been shown to predict user preference for different game design elements [75,77] and has been used to inform many gameful systems [3,19,25,53]. Thus, developing models to predict user preference for different persuasive strategies based on their Hexad user types holds value for designing personalized gameful systems.

To investigate how to tailor persuasive gameful systems to the six Hexad gamification user types (achiever, socialiser, philanthropist, free spirit, disruptor, and player), we conducted a large-scale study of 543 participants using Amazon's Mechanical Turk to examine how people of different user types respond to the ten commonly used persuasive strategies (competition, simulation, self-monitoring and feedback, goal setting and suggestion, customization, re- 
ward, social comparison, cooperation, personalization, and punishment). We employed Structural Equation Modeling [28] to model how people of different user types respond to or prefer various persuasive strategies implemented in storyboards. Our results reveal that an individual's user type predicts their preference for and the persuasiveness of different persuasive strategies. For example, people scoring high for the player user type tend to be motivated by competition, comparison, cooperation, and reward while disruptors can be demotivated by punishment, goal setting, simulation, and self-monitoring. Any persuasive strategies work for Socialisers, motivating them to adopt healthy behaviours, and thus they are generally the most responsive to persuasion. Our findings could guide designers in deciding on the best persuasive strategy to use and the ones to avoid when designing persuasive gameful systems targeting people of different user types.

Our work contributes to the fields of persuasive and gameful design in Human-Computer Interaction (HCI) in four ways. First, we reinforce the need for personalizing persuasive gameful systems by revealing that different user types respond differently to distinct persuasive strategies. Second, we establish that the Hexad user types is an important tool for personalizing gameful persuasive systems and selecting appropriate persuasive strategies. Third, we compare the effectiveness of individual strategies for the user types and develop guidelines for designing persuasive gameful health applications that appeal to a broad audience and a particular user type. Finally, we provide qualitative insights to explain why distinct strategies may motivate behaviours for people belonging to a particular user type and demotivate others. Our study is a first step towards developing models and guidelines for personalizing to the Hexad user types.

\section{RELATED WORK}

\section{Persuasive Strategies}

Persuasive systems tend to influence and encourage the user to adopt new behaviours and change undesirable behaviour [22]. This is usually done by employing techniques that are meant to persuade the user to change their behaviour, which are known as persuasive strategies. There are many persuasive strategies listed in the extant literature, such as the collections of seven strategies by Fogg [22] and 28 strategies by Oinas-Kukkonen and Harjumaa [34]. However, for this work, we have chosen to investigate ten persuasive strategies that are commonly employed in persuasive health applications [46] and that have been investigated in previous studies $[61,62]$ :

- Competition: Allows users to compete to perform the desired behaviour.

- Simulation: Provides the means for a user to observe the cause-and-effect linkage of their behaviour.

- Self-monitoring and Feedback: Allows people to track their own behaviours, providing information on both past and current states.
- Goal setting and Suggestion: Requires users to set a clear behaviour goal and recommend certain actions (to users for achieving the desired goal during system use).

- Customization: Allows users to adapt a system's contents and functionalities to their needs and choices.

- Reward: Offers virtual rewards to users for performing the target behaviour.

- Social Comparison: Provides a means for the user to view and compare their performance with the performance of other users.

- Cooperation: Requires users to cooperate (work together) to achieve a shared objective and rewards them for achieving their goals collectively.

- Personalization: Offers system-tailored contents and services based on the user's needs and characteristics.

- Punishment: Penalizes the user for not performing the desired behaviour or reaching their goal (such as removing acquired rewards or other of the user's possessions.)

Several persuasive gameful systems have employed these persuasive strategies to motivate risky health behaviour change. For example, Thinking Not Drinking: A SODAS City Adventure [69] is a persuasive gameful intervention for preventing unhealthy alcohol use. Thinking Not Drinking employs goal-setting and suggestion, personalization, simulation, reward, and social comparison to motivate behaviour change. Each game session begins with skillspecific goals that a player must accomplish before progressing - goal-setting. The game trains players on how to overcome peer pressure (social comparison) and avoid alcohol abuse. The game character is tailored to reflect the age, gender, and demographic background of the target audience - personalization. Players witness simulated consequences of their alcohol-related decision in the game simulation. Finally, to reinforce behaviours, players are rewarded. For a detailed review of persuasive health interventions and strategies employed, see Orji et al. [60].

\section{The Hexad User Types Model}

The Hexad [77] is a gamification user types model created to capture user's motivations and different styles of interaction with gameful systems. It proposes six user types, which are personifications of people's intrinsic and extrinsic motivations, as defined by self-determination theory $[14,68]$ :

- Philanthropists are motivated by purpose. They are altruistic and willing to give without expecting a reward.

- Socialisers are motivated by relatedness. They want to interact with others and create social connections.

- Free Spirits are motivated by autonomy and freedom to express themselves and act without external control. They like to create and explore within a system.

- Achievers are motivated by competence. They seek to progress within a system by completing tasks, or prove themselves by tackling difficult challenges.

- Players are motivated by external rewards or incentives. They will do whatever to earn a reward within a system, independent of the type of the activity. 
- Disruptors are motivated by the triggering of change. They tend to test the system's boundaries and disrupt the system either directly or through others to force negative or positive changes. They like to push further.

The Hexad user types model has been used to inform many gameful systems $[3,19,25,53]$. Again, previous work has shown that the user types of individuals are correlated with their preferences for different game design elements [75,77]. However, this only reflects user's enjoyment of the system, not their persuasiveness; their ability to motivate desired behaviour changes. To the best of our knowledge, there is yet no study investigating the relationship between the Hexad user types and individual preferences for distinct persuasive strategies. Nevertheless, we hypothesize that this relationship exists because the user types represent different preferences when people interact with gameful systems, and we can expect the persuasive strategies to be more effective when they rely on the user's preferred interaction style. Therefore, this study aims to test this hypothesis.

\section{Personalized Persuasive Gameful Systems}

As mentioned before, most existing persuasive gameful applications take a one-size-fits-all approach, rather than tailoring their content and strategies to individual users or groups [61]. However, many researchers have pointed to the limitations and risks of the one-size-fits-all approach to persuasive gameful systems design, especially when aimed at motivating health behaviour $[6,43]$. On the other hand, a few persuasive gameful systems for behaviour change have been designed for a specific user or cultural group. For example, Khaled et al. [43] demonstrated the feasibility of tailoring a persuasive gameful system to the individual's cultural background by developing two versions of a game (one for the collectivist and one for the individualist culture) using persuasive strategies that were deemed appropriate for each group. Their evaluation showed that players were persuaded more by their tailored version. PLAY, MATE! [6] is a persuasive gameful system for motivating physical activity, which tailored the reward strategies by varying the time required to complete a task between novice and experienced players. Finally, Orji [57] [58] showed the efficacy of personalization in the design and evaluation of JunkFood Aliens, a gameful application for motivating healthy eating behaviour. JunkFood Aliens tailored reward and competition and investigated the effect of tailored, contra-tailored, and one-size-fits-all approaches. Their results showed that tailoring increases the efficacy of persuasive gameful applications and that the untailored approach could be detrimental to behaviour change. Other examples of personalized gameful systems can be found in other domains, such as education $[4,21,25,26,54,55,63,74]$, social computing [79], crowdsourcing [20], and assisted living [23].

In most of these initial examples investigating how persuasive gameful systems for behaviour change can be tailored to increase their effectiveness, the choice of persuasive strategy employed has not been a source of tailoring. This is probably because only a few empirical research exists to guide the tailoring of strategies. For example, Orji et al. [61] established a relationship between an individual's personality traits and their preference for the ten persuasive strategies commonly used in gameful systems.

\section{STUDY DESIGN AND METHODS}

Our study was designed to investigate how to tailor persuasive gameful applications by examining the relations between the Hexad gamification user types and the perceived persuasiveness of the ten persuasive strategies for motivating risky health behaviour change, especially change of risky alcohol behaviour.

\section{Measurement Instrument}

To collect data for our study, we followed an established user study methodology that has been used in many CHI and related research (e.g., $[39,61,62])$. Specifically, we illustrated each of the persuasive strategies in a storyboard, which showed a character and their interactions with a persuasive gameful system for promoting change of unhealthy alcohol behaviours. The ten storyboards were adopted from a previous study where they have been used and validated [61]. The storyboards were drawn by an artist and were based on storyboard design guidelines by Truong et al. [78]. Implementing the strategies in storyboards makes it easier to elicit responses from diverse populations because storyboards provide a common visual language that individuals from diverse backgrounds can read and understand [47]. Moreover, storyboards have been shown to be effective at depicting strategies in previous research $[9,61,62]$. The implementations closely imitated how the strategies are operationalized in existing persuasive gameful systems from the literature $[36,37,69]$. We evaluated and iteratively refined the storyboards following three expert discussions: the first two with HCI and persuasive technology experts and the last one with an expert in rhetoric and narrative in games. Figure 1 shows an example of one of the storyboards illustrating the punishment strategy. The remaining storyboards are provided in the supplementary material.

To elicit feedback on the persuasiveness of the strategies, each storyboard was followed by a validated scale for assessing perceived persuasiveness. The scale was adapted from Drozd et al. [17] and has been used in other persuasive technologies research $[9,57,61,62]$. The scale consists of four questions: i) "The system would influence me."; ii) "The system would be convincing."; iii) "The system would be personally relevant for me."; iv) "The system would make me reconsider my alcohol drinking habits." The questions were measured using participant agreement with a 7point Likert scale ranging from " 1 = Strongly disagree" to "7 = Strongly agree". We also included open-ended questions that allowed participants to provide qualitative comments to justify their ratings of each strategy. Prior to assessing the persuasiveness of the strategies, we ensured that the participants understood the strategy depicted in each storyboard by asking them two comprehension questionsfirst, to identify the illustrated strategy from a list of 10 


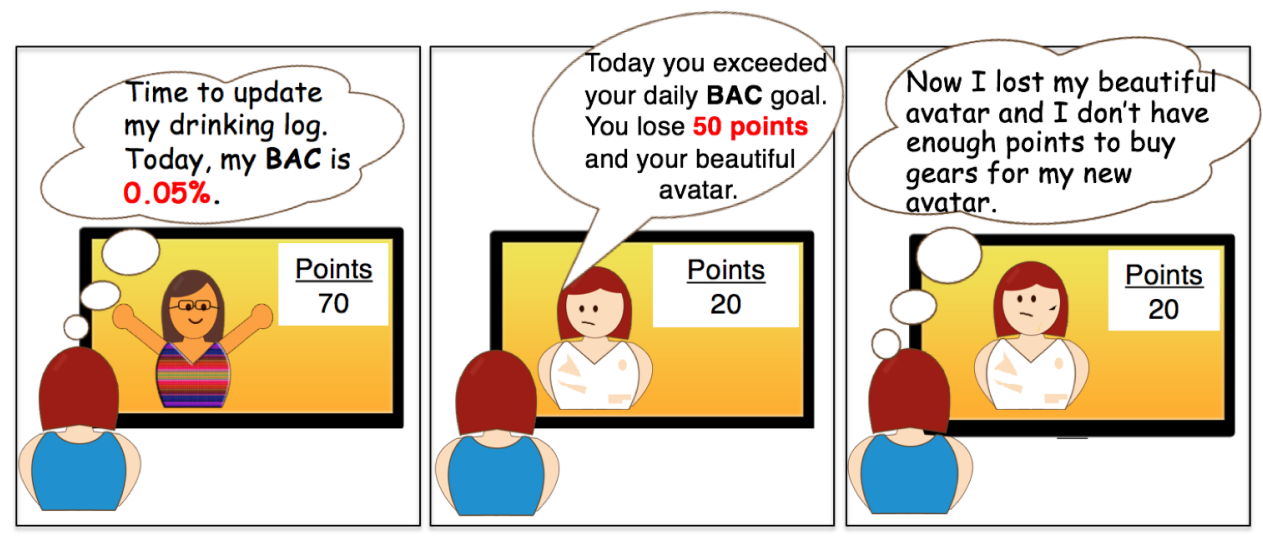

Figure 1. Storyboard illustrating the punishment persuasive strategy.

different strategies ("What strategy does this storyboard represent?"); and second, to describe what is happening in the storyboard in their own words ("In your own words, please describe what is happening in this storyboard"). We also included 24 items for assessing the Hexad gamification user types [77] and questions for assessing the participants' demographic information and drinking behaviours.

\section{Data Collection}

We recruited participants from Amazon's Mechanical Turk (AMT). We used AMT for two main reasons: first, AMT is an accepted method of gathering users' responses and has been used by many CHI studies $[5,35,39,61,62]$; and secondly, we needed a large participant sample from a diverse audience for our study. AMT allows access to a global audience at a relatively low cost, and ensures efficient survey distribution and high quality results $[8,51]$. Thus, AMT is suitable for this study which investigates technology preferences across a broader population in line with the type of tests that AMT is good at handling [5].

To eliminate possible storyboards ordering bias, we used the page randomization functionality provided by SurveyMonkey to rotate and vary the ordering of the storyboard for each participant. Before the main studies, we conducted two pilot studies to test the validity of our instruments. The first pilot study was conducted on 10 random students recruited from a Canadian University and the second on 10 participants from AMT.

\section{Participants' Demographic Information}

A total of 543 responses were included in this analysis, after filtering out incomplete responses and incorrect responses to comprehension and attention-determining questions [51]. Our participants were at least 18 years of age at the time of data collection, consumed or had consumed alcohol at some time, and read and understand English well. Participation required approximately 30 minutes. Participants were paid USD $\$ 2.00$ each; the payment rate is within the range of standard rate for similar tasks in AMT and in line with the study ethics approval. In general, we had a relatively diverse population in terms of gender, age, education level attained (see Table 1). Our participants came from the USA, India, Canada, and other countries.

\begin{tabular}{l|l}
\hline \multicolumn{2}{c}{ Total Participants = 543 } \\
\hline Gender & $\begin{array}{l}\text { Females (40\%), Males (59\%), Trans (1\%), } \\
\text { Others (0\%). }\end{array}$ \\
\hline Age & $\begin{array}{l}18-25(16 \%), 26-35(50 \%), 36-45(18 \%), \\
\text { Over 45 (15\%). }\end{array}$ \\
\hline Education & $\begin{array}{l}\text { Less than high school (1\%), High school } \\
(22 \%), \text { College diploma (14\%), Bachelor's } \\
\text { degree (44\%), Master's degree (17\%), } \\
\text { Doctorate degree (1\%), Others (2\%). }\end{array}$ \\
\hline Ethnicity & $\begin{array}{l}\text { Black/Non-Hispanic (6\%), Native Ameri- } \\
\text { can/American Indian (2\%), Asian/Pacific } \\
\text { Islander (24\%), Hispanic (6\%), Caucasian } \\
(59 \%), \text { Multi-Ethnic (3\%), Others (1\%) }\end{array}$ \\
\hline
\end{tabular}

Table 1. Participants' demographic information.

\section{DATA ANALYSIS}

To examine the relations between the gamification user types and the persuasiveness of the 10 commonly employed persuasive strategies used in persuasive gameful system design, we used several well-known analytical tools and procedures. We summarize the steps taken to analyze our data in this section:

1. We validated that our storyboards correctly depicted the intended strategy using a chi-squared test [29].

2. We determined the suitability of our data for analysis using the Kaiser-Meyer-Olkin (KMO) sampling adequacies test and the Bartlett Test of Sphericity [41].

3. Next, we employed the Partial Least Square (PLS) Structural Equation Modeling (SEM) (PLS-SEM) [66] to create models showing the relations between the gamification user types and the persuasiveness of the strategies (Figure 2). SEM is a recommended approach for modeling of relationships between variables [44]. We used SmartPLS 3 [66] for developing the models.

4. Finally, we used thematic analysis to identify and analyze qualitative comments provided by the participants in support of their quantitative score.

\section{Storyboard Validation}

To ensure that participants understood the strategy depicted in each of the storyboards, we ran chi-squared tests on the 
participants' responses to the multiple-choice questions that required them to identify the represented strategy for each of the storyboards. The results for all the strategies were significant at $p<0.0001$. This shows that our participants understand the storyboards and that the storyboards successfully depicted the intended strategies $[61,62]$.

\section{Measurement Validation}

To determine the suitability of our data for further analysis, we ran the Kaiser-Meyer-Olkin (KMO) sampling adequacies test and the Bartlett Test of Sphericity. The KMO was 0.95 , well above the recommended value of 0.6. The Bartlett Test of Sphericity was statistically significant $\left(\chi^{2}(780)\right.$ $=28412.672, p<0.0001)$. These results show that our data were suitable for further analysis [39, 45].

We report here the common set of indices for model validity and reliability in PLS-SEM. The required criteria for the PLS-SEM validity and reliability were satisfied. Indicator reliability can be assumed because Cronbach's $\alpha$ and the composite reliability that analyze the strength of each indicator are all higher than their threshold value of 0.7 [12]. We checked the data for both convergent and discriminate validity. All constructs have an AVE (which represents the average variance extracted by the variables from its indicator items) above the recommended threshold of 0.5 [12]. The heterotrait-monotrait ratio of correlations (HTMT) [28] were all below the recommended limit of 0.9 .

In the next subsection, we present the results showing the relationship between the gamification user types and the 10 persuasive strategies.

\section{The Structural Model}

The structural models show the relations between the gamification user types and the persuasiveness of individual strategies (see Figure 2). The latent variables used in the model were the participant's scores for each Hexad user types and the perceived persuasiveness of each strategy. To measure the strength of the relationships between the variables in the structural models, we calculated the path coefficient $(\beta)$, and the significance of the path coefficient $(p)$ [27], which are the established criteria. Path coefficients measure the influence of one variable on another. The individual path coefficients $(\beta)$ and their corresponding level of significance $(p)$ obtained from our models are summarized in Table 2.

\begin{tabular}{|c|c|c|c|c|c|c|}
\hline Factors & PLA & PHI & DIS & FRE & SOC & $\mathrm{ACH}$ \\
\hline $\begin{array}{l}\text { Goal-setting and } \\
\text { Suggestion }\end{array}$ & - & - & -.15 & - & .20 & - \\
\hline Competition & .26 & - & .11 & - & .25 & - \\
\hline Comparison & .14 & - & - & - & .28 & - \\
\hline Cooperation & .13 & - & - & - & .29 & - \\
\hline Customization & - & - & .14 & - & .31 & - \\
\hline Reward & .15 & - & - & - & .33 & - \\
\hline Punishment & .11 & - & -.12 & - & .32 & - \\
\hline Personalization & - & - & -.15 & .13 & .17 & - \\
\hline Simulation & - & .18 & -.15 & & .17 & - \\
\hline $\begin{array}{l}\text { Self-monitoring } \\
\text { and Feedback }\end{array}$ & - & - & -.14 & - & .19 & - \\
\hline \multicolumn{7}{|c|}{$\begin{aligned} \text { PLA } & =\text { Player, } \mathrm{PHI}=\text { Philanthropist }, \mathrm{DIS}=\text { Disruptor, SOC } \\
& =\text { Socialiser, FRE }=\text { Free Spirit }, \mathrm{ACH}=\text { Achiever }\end{aligned}$} \\
\hline
\end{tabular}

Table 2. Standardized path coefficients and significance of the relationships. Bolded coefficients are $p<.001$, non-bolded are $\mathbf{p}<.05$, and '-' represents non-significant coefficients.

\section{Relationships Between Gamification User Types and Persuasive Strategies}

The results from the structural model show that participants' user type as identified by the Hexad scale influences the persuasiveness of individual strategies (see Table 2). In this section, we discuss and compare the persuasiveness of the strategies for people having different user types.

\section{Goal-setting and Suggestion}

The goal-setting and suggestion strategy is derived from the goal-setting theory $[48,49]$, which posits that setting behaviour goals and receiving suggestions on how to achieve the goals promotes behaviour performance. Our results show that goal setting is only a significant motivator for people high in socialiser tendencies ${ }^{1}(\beta=.20, p<.001)$. It is possible that socialisers perceived the suggestions as a form of assistance, which has been previously shown to be valued by socialisers [75,77]. Some of the reasons for socialisers' high preference for goal setting and suggestion as highlighted in the qualitative comments to justify their ratings include that it provides opportunity for selfunderstanding and self-motivation; makes people focused, committed to their goal, responsible, and conscious (of their behaviour), as shown in the following comments:

"Setting goals and working toward them to achieve them is self-motivating for me and provides opportunity for selfunderstanding" $[\mathrm{P} 411]^{2}$. "This would make me more aware of how much I was drinking. In this sense, it would make me more responsible for my drinking” [P19]. “...I

\footnotetext{
${ }^{1}$ In the 7-point Likert Hexad scale, a participant is categorized as high in a particular user type if they score 4.5 and above, while 2.5 and below are classified as low.

${ }^{2}$ Quotes from participants are included verbatim throughout the paper, including spelling and grammatical mistakes.
}

Figure 2. PLS-SEM model structure. 
like this the best so far, suggestive, but the user is still in control" [P255].

On the other hand, goal-setting and suggestion could demotivate behaviour for people high in disruptor tendencies $(\beta=$ $-.15, p<.001)$ and would have no significant effect on people with high achiever, philanthropist, player, and free spirit tendencies. The low preference from disruptors can be explained by the fact that they enjoy pushing boundaries [77], which is more difficult if one has pre-established goals. Additional reasons for this low preference for goal setting and suggestion include its tendency to be boring, not incentivizing, intrusive, and potentially backfiring:

"Seems very boring without incentives" [P24]. "Since the system tracks how I am doing in relation to my goal, I could set my goal high which would allow me to drink more alcohol" [P181]. "It doesn't really give any incentive, so there is no genuine motivation for change" [P347].

These comments suggest that goal-setting would need complementary strategies such as rewards (badges, points) to incentivize users and motivate some people.

\section{Competition}

Competition builds on the assumption that individuals will be motivated to perform the desired behaviours if they are allowed to compete with others [22]. Our results show that competition would motivate behaviours for people with high player, socialiser, and disruptor tendencies: $(\beta=.26$, $p<.001)(\beta=.25, p<.001)$ and $(\beta=.11, p<.05)$, respectively. This is in line with prior research [77]. Some reasons given to justify the preference for competition from players, socialisers, and disruptors include that it reinforces and encourages behaviours; makes behaviour fun and appear easier to do; and makes people committed and focused:

"The kind of positive reinforcement it gives through competition with other would certainly influence me to reconsider my drinking habits" [P112]. "Competition makes me committed and focused on my goal" [P352]. "Behaviour change seems easier when you're in a competition" [P501]. "A little healthy competition will inspire the user to continue making healthy choices" [P274]. "The competition aspect of this application is highly useful. It is very encouraging and would help me to change my intake drastically" [P42].

Competition is not a significant motivator for people high in achiever, free spirit, and philanthropist tendencies. Some reasons to justify their low preference for competition include its perception as having the potential to reduce selfesteem, cause anxiety and depression, encourage body shaming, demotivate and trivialize the benefit of behaviour. This is to be expected because achievers, free spirits, and philanthropists user types tend to focus on their own advancement within the system or in helping others, rather than on comparing their performance with others [77].

"...it would also make me feel like a failure and likely increase my depression and low self-esteem" [P119]. "I'm not sure direct competition is a good idea. It makes winners and losers and being labeled a loser generally doesn't lead to better behaviour" [P276]. "...it may demotivate a person who can never win to quit trying" [P533]. "Would increase anxiety and negative feelings that cause me to drink, knowing I have pressure to succeed in the contest" [P429].

\section{Personalization and Customization}

Personalization and customization are two distinct persuasive strategies, however, we discuss them together because they both aim to achieve the same objective of tailoring systems, although with different approaches [62]. In customization, system tailoring is done by the user, while personalization is mostly system-controlled [61]. Previous research has suggested that allowing users to do the tailoring themselves increases the system's effectiveness because it gives users a strong sense of autonomy and control $[61,62,73]$. Our results show that these two strategies are perceived differently by our participants. Customization would motivate behaviour for people higher in socialiser and disruptor tendencies $(\beta=.31, p<.001)$ and $(\beta=.14$, $p<.05$ ), while it is not significant for achievers, philanthropists, free spirits, and players. Some reasons for the high preference from socialisers and disruptors include the fact that it gives users a sense of control, choice, and personal touch; it increases system appeal, relevance, ease of use; and it makes the system engaging and personable, which can particularly satisfy the disruptors' need to be in control and to modify the system to their needs and desires [77].

"It makes it more personal" [P15]. "It is good to be able to customize to my own personal preferences and ensures the system is relevant to me" [P125]. "Customization enables me use the application more easily. The easier the application is to use the easier it becomes to track my daily alcohol intake and stay motivated" [P467]. "I think it is great to have more control over your health systems" [P74]. "It would make it feel more like "mine" [P2] "By allowing customization, the system draws one in and makes it more likely the person will be 'engaged' in the task" [P90].

On the other hand, personalization is a significant motivator for people high in socialiser and free spirit tendencies $(\beta=$ $.17, p<.001)$ and $(\beta=.13, p<.05)$, respectively. Some reasons for this high preference for personalization from socialisers and free spirits include its potential to increase system's usefulness, relevance, credibility, and the user's confidence and trust in the system:

"Personalized feedback makes the information more relevant and memorable" [P52]. "I love the personalization idea! It draws the user in making them feel it's just for them, thereby making it more likely the user will follow the guidelines" [P200]. "Because it's personalized, it's better, unique, valuable, and more useful to each individual person using it" [P112]. "I would be a lot more confident in the app and motivated if I knew it was personalized to me..." [P15]. "I would feel that this is based on good scientific data, and trust its recommendations" [P34]. 
Personalization would demotivate behaviour for people high in disruptor tendencies $(\beta=-.15, p<.05)$ and would do nothing for achievers, philanthropists, and players. This is understandable, considering that disruptors are motivated by having power to influence the system [77], and a system that automatically personalizes the content could decrease their sense of power.

\section{Reward}

Reward is commonly used because of its ability to incentivize users. In line with the common believe, reward is one the strategies that is not negatively associated with any of the user types. As shown in Table 2, reward is perceived as significantly positive by people high in socialiser and player tendencies: $(\beta=.33, p<.001)$ and $(\beta=.15, p<.001)$, respectively, which aligns with prior research $[75,77]$. Some reasons given for this high preference for reward from socialisers and players include that it makes behaviour fun; reinforces behaviour; boosts user's confidence; engages, incentivizes, and gives people something to look forward to:

"Rewards boost confidence and also motivates me" [P59]. "I think the reward program is a great way to hold the attention of most people as long as the system regularly has new rewards for the users" [P419]. "...getting rewards in the game for meeting my goal makes me try hard" [P9]. "The system is a fun way to help one not to drink too much and to earn points" [P214]. "A reward system is an additional motivator to help the user reach their goals and get invested in the app" [P67].

Reward is not a significant motivator for people high in achiever, philanthropist, free spirit, and disruptor tendencies. Some reasons for the low preference for reward include its tendency to misrepresent and trivialize the benefit of behaviour and be perceived as childish:

"Offered rewards are easily mistaken to be the benefits of health behaviour for individual users, it may be misinterpreted" [P126]. "It makes me feel like a 5-year old" [P19]. "Rewarding good behaviour can be effective, but I would need to know what the points represent or if they are redeemable for anything" [P505].

The last comment suggests that the effectiveness of rewards for certain people may be dependent on what the rewards can be used for (i.e., the tangible value of the reward $[64,65])$. This low preference for rewards from achievers, philanthropists, free spirits, and disruptors might also be explained by the fact that these user types usually prefer to be intrinsically motivated to interact with a system rather than extrinsically [77]. Therefore, offering external rewards might lead them to feel that their intrinsic motivation to adopt the healthier behaviour is being undervalued.

\section{Punishment}

In line with reinforcement theory [71], some persuasive gameful systems use punishment to discourage undesired behaviour and motivate desired behaviours. It has been argued that punishment may not be as effective for promoting behaviour change as reward [13,61]. However, our results show that similar to reward, punishment would motivate behaviour for people high in socialiser and player tendencies: $(\beta=.32, p<.001)$ and $(\beta=.11, p<.05)$. Some reasons given to justify this high preference for punishment from socialisers and players include the potential of punishment to reinforce behaviour and make it fun; incentive users; make them work hard; and keep them at alert and focused:

"Small punishments could reinforce the idea that I need to drink less and improve my health" [P522]. "Having something to lose would give me an extra kick and influence me to achieve the goal" [P212]. "Getting and losing points would motivate me to try hard and lower my drinking" [P121]. "Penalty will make me stay alert and focused of my goals" [P100].

Punishment is not a significant motivator for achievers, philanthropists, and free spirits and could demotivate behaviour performance for people high in disruptor tendencies $(\beta=-.12, p<.05)$. This lack of preference from disruptor stems from them usually seeking to push boundaries without fear of serious consequences [77], which would be impossible if they were expecting a punishment for lack of compliance. Some explanations for the low preference for punishment include its tendency to frustrate, discourage, and make some people feel bad:

"I feel like punishment is the wrong strategy. People often drink too much because they're prone to depression or feel like failures in various areas of their life. A system that reinforces that seems counterproductive" [P106]. "The moment I get punished for something, I would stop using the application" [P195]. "I think the punishment would make me feel worse about myself and not encourage me to do better next time" [P43].

\section{Social comparison}

Social comparison provides mechanisms that allow users to view and compare their performance with that of their peers. However, there is no winning or losing involved, differentiating it from competition [61]. Our results show that social comparison would motivate behaviour change for people high in socialiser and player tendencies $(\beta=.28$, $p<.001)$ and $(\beta=.14, p<.05)$, respectively. This is in line with prior research [77]. Some reasons for this high preference from socialisers and players include that social comparison provides an indirect support network and role models, help build confidence, and stay accountable.

"It creates some indirect support network but it's not a competition; you would never feel alone in the process." [P189]. "It would provide a type of support group so I can compare my goal with others and see how they are doing. I am likely to try harder when I see that my friends are achieving their goals" [P1]. "I wouldn't want to be a bad example and role model for my peers, so will definitely make me work harder" [P159]. "It could help build confidence by connecting with others that're also struggling to overcome the same issue" [P117]. 
Social comparison is not a significant motivator for people high in achiever, philanthropist, free spirit, and disruptor tendencies. Similar to competition, this can be explained because these user types describe people who enjoy progressing by themselves instead of comparing their performance with others. Some reasons for this low preference for social comparison include its tendency to be intrusive; breach privacy; backfire; make people feel guilty, ashamed, and inferior.

"I don't like having my drinking habits compared to others. It just makes me feel inferior" [P227]. "WOW, that's a breach of privacy, will never use it" [P501]. "It seems intrusive" [P11]. "This might back fire, promote more drinking if everyone drinks more" [P99]. "I'd feel embarrassed if I didn't do as well as my friends" [P467]. "The system offers a benchmark that may be skewed if other users are also struggling with their goals" [P389]. "Runs a high risk of causing self-shame and guilt, which can increase problem drinking behaviours" [P12].

\section{Cooperation}

A system can motivate by providing users opportunities to work with others cooperatively [56]. Our results show that cooperation would motivate behaviour change for people high in socialiser and player tendencies: $(\beta=.29, p<.001)$ and $(\beta=.13, p<.05)$, respectively. Some reasons for this high preference from socialisers and players include that cooperation provides opportunity for people to partner, support one another, lean on and encourage each other, stay accountable, responsible, and feel not alone:

"I would enjoy partnering with and helping someone else meet their goals" [P368]. "...Having another person to lean on and help could help yourself as well" [P475]. "Working with friends towards a goal would give you a support system" [P507]. "Working together make someone feel less lonely in the journey of behaviour change" [P261]. "I like that it makes me accountable to other people. I wouldn't want to let them down" [P96].

Cooperation is not a significant motivator for people high in achiever, philanthropist, free spirit, and disruptor tendencies. Once more, this can be explained by the preference of people that score high in these user types for progressing on their own rather than by comparing their progress with others (even if the comparison is not explicitly encouraged by the system) [77]. The main negative comment against cooperation is its tendency to cause shame and guilt, unnecessary pressure, anxiety, frustration; and is not privacy preserving.

"I would not want to be pressured by my friends to control my drinking to earn bonus points" [228]. "I think being unfairly matched in a team effort is discouraging and frustrating" [P256]. "Cooperation and team work doesn't help for an individual based problem" [P18]. "...providing strong feelings of guilt and shame, probably driving me into a downward spiral' [P199].
Self-monitoring

Our results show that self-monitoring and feedback is significantly associated with people who are high in socialiser tendencies $(\beta=.19, p<.001)$. Some reasons for this preference include its tendency to reveal problem behaviours, show progress, create awareness, offer useful feedback, foster reflection and make users accountable:

"I used to be an alcoholic but I've been sober for 9 months. Something like this would have made me feel a sense of progress and achievement, rather than just a timeline (i.e., " $x$ " amount of months)" [294]. "Being able to log progress would help greatly with accountability and selfevaluations" [P542]. "Personal competition is somewhat sufficient and better than interpersonal competition when it comes to healthy living" [P78]. "Looking back on your history might be very revealing and eye opening to some people" [P457]. "This system would help me be aware of my consumption and reflect on it..." [P359]. "The system would be able to provide me useful information that would inform my future drinking decisions" [P469].

Self-monitoring and feedback would demotivate behaviour for people high in disruptor tendencies $(\beta=-.14, p<.05)$. This can be explained by the disruptors' tendency to act more out of creativity and freedom, rather than following a strictly monitored path.

\section{Simulation}

"An application can motivate people to change their behaviour by providing a way for them to observe the cause and effect linkage of their behaviours" [61]. In line with this proposition, our results show that simulation is a significant motivator for people high in philanthropist and socialiser tendencies: $(\beta=.18, p<.001)$ and $(\beta=.17, p<.001)$. This is understandable considering that philanthropists are motivated by the achievement of meaningful goals, and simulation can help them realize the implications and consequences of their efforts. Some other reasons for this preference for simulation from philanthropists and socialisers include that it offers practical advice; real and concrete information; raises awareness; provoke deep reflection; and allow users to visualize possible behavioural outcomes:

"I think that using the application makes the behaviour real and concrete for me. Hence, the consequences and benefits become real as well and motivating" [P188]. "Learning about the negative effects of dangerous behaviour regarding alcohol could really allow for effective reflection on past less-than positive alcohol consumption" [P362]. "This gives practical advice" [P421].

Simulation is not a significant motivator for people high in achiever, free spirit, and player tendencies and it would demotivate behaviour for disruptors $(\beta=-.15, p<.001)$. This is probably because people high in these user types tend to focus more on the immediate motivation for doing something (achievement, creativity, or rewards) [77] rather than the long-term meaning of their actions. 
Summary of Hexad Gamification User Types and Persuasive Strategies

In summary, socialiser and player emerged as the user types that are most motivated by the persuasive strategies overall. Socialiser is positively associated with all the strategies, while player is positively associated with five (out of the ten strategies) and negatively associated with none. Achiever and disruptor emerged as the least responsive user types. Disruptor is negatively associated with most of the strategies, while achiever shows no significant relation with any of the strategies.

\section{DISCUSSION}

In this section, we discuss how our findings can be used in designing persuasive gameful systems to appeal to both a broader audience and to be tailored to a particular user group based on their user type.

\section{Designing to Appeal to a Broad Audience}

Competition emerged as the most persuasive of all the strategies from our results. It appeals to three of the user types (player, socialiser, and disruptor) and does not negatively influence any user types. Therefore, to appeal to a broad audience, persuasive gameful systems designers should provide a mechanism that allow users to compete to perform the desired behaviour. Game mechanics such as leaderboard, status, envy, and countdown ${ }^{3}$ could be used to show player's performance relative to others; allowing them to compete to motivate them to work harder and perform better than others in line with the competition.

Our results also show that cooperation is perceived as positive by players and socialisers and does not impact negatively on any user type. Therefore, we recommend that to appeal to a broad user population, persuasive gameful systems designers should implement mechanisms to allow users to work together (collaborate) to motivate desired behaviour performance. This suggest that cooperative internet-based play for health (i.e., social games) would appeal to a board population [62]. Thus, mechanics such as communal discovery, social fabric of games, viral game mechanics, and companion gaming could be used to create a sense of community and make the players work together to achieve the desired health behaviour.

Similarly, our results show that social comparison is perceived as positive by socialiser and player and does not impact on any of the user types negatively. Therefore, to appeal to a broad audience, persuasive gameful systems should be designed to allow users to view and compare their performance with that of others. Game mechanics such as leaderboard, status, envy, and countdown could be used to show players how they are doing relative to others. This mechanics do not have to involve winning or losing (overt competition) to be effective.

\footnotetext{
${ }^{3}$ For detailed definitions of the game elements and the mapping of the persuasive strategies to some common game mechanics, see Orji et al. [62].
}

Our results also show that reward is perceived as positive by player and socialiser and is not negatively associated with any user type. Therefore, to appeal to a broad audience, we recommend that persuasive gameful systems should employ mechanisms that reward users to motivate them to perform the desired behaviours. Game mechanics that suggest some kind of incentive such as bonuses, points, free lunch, virtual items, reward schedules, lottery, physical goods can be applied to operationalize reward and motivate desired health behaviour performance.

Our qualitative comments reveal that one common weakness of the social influence strategies - competition, social comparison, and cooperation - is their tendency to promote body shaming and interfere with an individual's privacy. Therefore, we recommend that care should be taken when applying these strategies to preserve user's privacy and reduce the tendency of application-induced body shaming. Designers could anonymize behaviour data or present performance data as a percentage of an individual goal. This would be a good privacy-preserving and shamereducing alternative to displaying actual behaviour data. Another common weakness of these strategies is that their effectiveness could be dependent on the social circle. For example, a social comparison circle consisting of heavy drinkers could backfire by encouraging one to drink more because his/her benchmark are heavy drinkers. Therefore, designers should apply some caution when employing the social influence strategies in designs to reduce the likelihood of downward and negative social influence. Designers can pre-screen users to understand how to distribute them across groups to ensure effective social circle for comparison, competition, and cooperation. Designers can also include mechanisms that allow for both withingroup and between group social influence [61].

\section{Designing for People with a Specific User Type}

Designing for a broad audience is a common practice; however, research has advocated that persuasive experiences should be tailored to increases their efficacy [42,57,59]. Our results reveal opportunities to personalize persuasive gameful design by tailoring the strategies to the user types.

For example, our results show that simulation is the only strategy that is perceived as significantly positive by people with high philanthropist tendencies, possibly because it helps them understand the long-term meaning of their efforts. Hence, they are more likely to be motivated by a system employing this strategy. Therefore, we suggest that when designing to specifically appeal to people who are high in philanthropist tendencies, persuasive gameful systems should be designed to show the choice-andconsequences linkage and projected outcomes of an individual's health behaviour. Game elements such as achievements, epic meaning, behaviour momentum, blissful productivity, and urgent optimism that structure play and give players an idea of how their behaviour will impact their lives could be used to create a simulated experience of the real-world behaviour within the context of play [61]. 
For people with high free spirit tendencies, our results show that personalization is the only strategy that significantly appeals to them. Therefore, we suggest that when designing to specifically appeal to people who are high in free spirit tendencies, designers should tailor the system contents and functionalities using system-controlled tailoring. Game mechanics such as cascading information theory, epic meaning, and privacy could be used to create a sense of personalized contents and personal relevance to motivate free spirit to perform the desired health behaviour.

Regarding disruptors, who are motivated by change and control [77], our results show that they are persuaded by customization and competition only. This is understandable considering that customization allows the user the flexibility to change and alter the system to suite their preference, while competition challenges them to push boundaries and subdue others in line with their inherent characteristics. Therefore, persuasive gameful systems tailored for disruptors can effectively employ mechanisms that suggest customization and competition. For example, the game mechanics discovery, shell games, and epic meaning could work well because they can be used to create an illusion of choice and control, which customization provides.

Our findings show that people high in achiever tendencies are less likely to be motivated to adopt healthy behaviours using any of the ten strategies studied. This is surprising; however, a possible explanation is that the most commonly employed persuasive strategies or their operationalization are not suitable for achievers. Therefore, persuasive gameful systems designers should explore achiever-oriented persuasive strategies. Another possible explanation is that persuasion may not work for everyone, there is a limit to what and who can be persuaded using the strategies [61]. Therefore, for achievers, persuasion may not be an effective approach for motivating behaviour change.

Finally, our findings reveal that socialiser, disruptor, and players are the three Hexad user types that predict most of the variability in the effectiveness of persuasive strategies. Socialiser and player are significantly and positively associated with most strategies, while disruptor is negatively associated with most strategies. Therefore, to achieve user type-driven tailoring, it is necessary to at least differentiate participants based on these three user types.

There are many ways our results can be used to tailor persuasive gameful systems. We have included only a few examples here to demonstrate that. Table 2 details the relations between the strategies and user types which could guide design choices for tailoring persuasive gameful systems. To identify an individual's user type for personalization, designers should follow the guideline specified in the Hexad framework [77]. Then, our results in Table 2 and the suggested guidelines can be used in deciding on the appropriate persuasive strategy and corresponding game mechanics to operationalize them in persuasive gameful systems to motivate desirable health behaviour.

\section{LIMITATIONS}

First, we used the self-reported persuasiveness of the strategies implemented in storyboards; the actual persuasiveness of the strategies may differ when implemented in a real application. Thus, we plan to evaluate the persuasiveness of the strategies in actual persuasive gameful systems. However, research has shown that players' responses to real games relates to their response to storyboard implementation [58]. Secondly, the persuasive strategies can be operationalized in designs; however, while our storyboards reflected the most common implementations from the literature, we were not able to explore different ways of implementing each strategy. Again, culture and personal attitude towards drinking may play an important role on persuasion and our study did not explore that. Finally, we conducted this study in the context of unhealthy alcohol behaviour change and we can claim applicability in other health behaviour domains due to the high-level nature of the storyboard depicting the strategies; however, we acknowledge that our results may not generalize and therefore should be applied with caution in other behaviour domains.

\section{CONCLUSION AND FUTURE WORK}

The current study investigated the relations between gamification user types and persuasive strategies for the first time in the literature. This paper makes an initial contribution to understanding how to tailor persuasive gameful systems to increase their efficacy based on how the user's responsiveness to persuasive strategies is determined by their user type. As a secondary objective, we provide qualitative insights based on users' comments to explain why distinct strategies may motivate behaviours for people belonging to a particular user type and demotivate others. Through our study, we uncovered the shortcomings of the untailored approach and presented design opportunities for designing persuasive gameful systems that appeal both to a broad audience and for tailoring to a particular user type. Our findings indicate that socialiser, disruptor, and player are the three gamification user types that predict most of the variability in the effectiveness of persuasive strategies and thus, must be taken into account to achieve user-type-driven tailoring. Our findings could guide designers in making informed choices on the strategies to employ and those to avoid when designing persuasive tailored gameful systems.

In the future, we plan to apply the guidelines in designing and evaluating the effectiveness of actual tailored persuasive gameful systems and to validate our findings across other health behaviour domains (e.g., discouraging drug use, risky sexual behaviour, and smoking) to investigate possible variability in the persuasiveness of the strategies. We hope to explore the role of culture and personal attitude towards drinking on the persuasiveness of the strategies.

\section{ACKNOWLEDGMENTS}

Many thanks to the NSERC Banting and the CNPq, Brazil, SSHRC (895-2011-1014, IMMERSe), NSERC (RGPIN418622-2012), CFI (35819), and Mitacs (IT07255) for funding our research. 


\section{REFERENCES}

1. Tuomas Alahäivälä and Harri Oinas-Kukkonen. 2016. Understanding persuasion contexts in health gamification: A systematic analysis of gamified health behavior change support systems literature.

International Journal of Medical Informatics 96: 6270. https://doi.org/10.1016/j.ijmedinf.2016.02.006

2. Ahmed Allam, Zlatina Kostova, Kent Nakamoto, and Peter Johannes Schulz. 2015. The effect of social support features and gamification on a Web-based intervention for rheumatoid arthritis patients: randomized controlled trial. Journal of Medical Internet Research 17, 1: e14. https://doi.org/10.2196/jmir.3510

3. Darius Ašeriškis and Robertas Damaševičius. 2017. Computational Evaluation of Effects of Motivation Reinforcement on Player Retention. Journal of Universal Computer Science 23, 5: 432-453. Retrieved from http://www.jucs.org/jucs_23_5/ computational_evaluation_of_effects

4. Gabriel Barata, Sandra Gama, Joaquim Jorge, and Daniel Gonçalves. 2017. Studying student differentiation in gamified education: A long-term study. Computers in Human Behavior 71: 550-585. https://doi.org/10.1016/j.chb.2016.08.049

5. Frank R. Bentley, Nediyana Daskalova, and Brooke White. 2017. Comparing the Reliability of Amazon Mechanical Turk and Survey Monkey to Traditional Market Research Surveys. In Proceedings of the 2017 CHI Conference Extended Abstracts on Human Factors in Computing Systems - CHI EA '17, 10921099. https://doi.org/10.1145/3027063.3053335

6. Shlomo Berkovsky, Mac Coombe, Jill Freyne, Dipak Bhandari, and Nilufar Baghaei. 2010. Physical activity motivating games: virtual rewards for real activity. In Proceedings of the International Conference on Human Factors in Computing Systems, 243-252. https://doi.org/10.1145/1753326.1753362

7. Martin Böckle, Jasminko Novak, and Markus Bick. 2017. Towards Adaptive Gamification: a Synthesis of Current Developments. In Proceedings of the $25^{\text {th }}$ European Conference on Information Systems (ECIS). Retrieved from http://aisel.aisnet.org/ecis2017_rp/11

8. M Buhrmester, T Kwang, and Gosling S. D. 2011. Amazon's Mechanical Turk A New Source of Inexpensive, Yet High-Quality, Data? Perspectives on Psychological Science 6, 1: 3-5. https://doi.org/doi: $10.1177 / 1745691610393980$

9. M. Busch, E. Mattheiss, M. Reisinger, R. Orji, P. Fröhlich, and M. Tscheligi. 2016. More than sex: The role of femininity and masculinity in the design of personalized persuasive games. PERSUASIVE 2016. https://doi.org/10.1007/978-3-319-31510-2_19
10. Joseph A Cafazzo, Mark Casselman, Nathaniel Hamming, Debra K Katzman, and Mark R Palmert. 2012. Design of an mHealth app for the selfmanagement of adolescent type 1 diabetes: a pilot study. Journal of Medical Internet Research 14, 3: e70. https://doi.org/10.2196/jmir.2058

11. Yu Chen and Pearl Pu. 2014. HealthyTogether: exploring social incentives for mobile fitness applications. In Proceedings of the Second International Symposium of Chinese CHI, 25-34. https://doi.org/10.1145/2592235.2592240

12. W. Wynne Chin. 1998. The Partial Least Squares Approach to Structural Equation Modeling. Modern Methods for Business Research 292, 2: 298-336.

13. Aubrey C. Daniels. 2000. Bringing Out the Best in People: How to Apply the Astonishing Power of Positive Reinforcement. McGraw-Hill.

14. Edward L Deci and Richard M Ryan. 1985. Intrinsic Motivation and Self-Determination in Human Behavior. Plenum, New York and London.

15. Tracy A. Dennis and Laura J. O’Toole. 2014. Mental Health on the Go: Effects of a Gamified Attention-Bias Modification Mobile Application in Trait-Anxious Adults. Clinical Psychological Science 2, 5: 576-590. https://doi.org/10.1177/2167702614522228

16. Sebastian Deterding, Dan Dixon, Rilla Khaled, and Lennart E Nacke. 2011. From Game Design Elements to Gamefulness: Defining "Gamification.” In Proceedings of the $15^{\text {th }}$ International Academic MindTrek Conference, 9-15. https://doi.org/10.1145/2181037.2181040

17. Filip Drozd, Tuomas Lehto, and Harri OinasKukkonen. 2012. Exploring perceived persuasiveness of a behavior change support system: A structural model. In Lecture Notes in Computer Science (including subseries Lecture Notes in Artificial Intelligence and Lecture Notes in Bioinformatics), 157-168. https://doi.org/10.1007/978-3-642-31037-9_14

18. E A Edwards, J Lumsden, C Rivas, L Steed, L A Edwards, A Thiyagarajan, R Sohanpal, H Caton, C J Griffiths, M R Munafò, S Taylor, and R T Walton. 2016. Gamification for health promotion: systematic review of behaviour change techniques in smartphone apps. BMJ Open 6, 10. https://doi.org/10.1136/bmjopen-2016-012447

19. Ahmed Fadhil and Adolfo Villafiorita. 2017. An Adaptive Learning with Gamification \& Conversational UIs: The Rise of CiboPoliBot. In Adjunct Publication of the $25^{\text {th }}$ Conference on User Modeling, Adaptation and Personalization - UMAP '17, 408-412. https://doi.org/10.1145/3099023.3099112 
20. Oluwaseyi Feyisetan, Elena Simperl, Max Van Kleek, and Nigel Shadbolt. 2015. Improving Paid Microtasks through Gamification and Adaptive Furtherance Incentives. In Proceedings of the $24^{\text {th }}$ International Conference on World Wide Web - WWW'15, 333-343. https://doi.org/10.1145/2736277.2741639

21. Richard Filipcik and Maria Bielikova. 2014. Motivating Learners by Dynamic Score and Personalized Activity Stream. In $20149^{\text {th }}$ International Workshop on Semantic and Social Media Adaptation and Personalization, 20-25. https://doi.org/10.1109/SMAP.2014.25

22. Brian J. Fogg. 2003. Persuasive Technology: Using Computers to Change What We Think and Do. Morgan Kaufmann. https://doi.org/10.1145/764008.763957

23. Martin Franke, Bianca Zimmer, and Thomas Schlegel. 2015. An Adaptive, Structural and Content Gamification Concept for Regulated Daily Routines. In Proceedings of the International Conference on Biomedical Electronics and Devices, 233-240. https://doi.org/10.5220/0005317802330240

24. Konstantinos Giannakis, Konstantinos Chorianopoulos, and Letizia Jaccheri. 2013. User requirements for gamifying sports software. In $20133^{\text {rd }}$ International Workshop on Games and Software Engineering (GAS), 22-26. https://doi.org/10.1109/GAS.2013.6632585

25. Borja Gil, Iván Cantador, and Andrzej Marczewski. 2015. Validating Gamification Mechanics and Player Types in an E-learning Environment. In Design for Teaching and Learning in a Networked World, LNCS vol. 9307. Springer, Cham, 568-572. https://doi.org/10.1007/978-3-319-24258-3_61

26. Carina S González, Pedro Toledo, and Vanesa Muñoz. 2016. Enhancing the Engagement of Intelligent Tutorial Systems through Personalization of Gamification. International Journal of Engineering Education 32, 1: 532-541.

27. Joe F. Hair, Christian M. Ringle, and Marko Sarstedt. 2011. PLS-SEM: Indeed a Silver Bullet. Journal of Marketing Theory and Practice 19, 2: 139-152. https://doi.org/10.2753/MTP1069-6679190202

28. Joseph F. Hair Jr, G. Tomas M. Hult, Christian Ringle, and Marko Sarstedt. 2016. A primer on partial least squares structural equation modeling (PLS-SEM). Sage Publications.

29. Sajanee Halko and Julie A Kientz. 2010. Personality and Persuasive Technology: An Exploratory Study on Health-Promoting Mobile Applications. In Persuasive Technology, 150-161. https://doi.org/10.1007/978-3-642-13226-1_16

30. Margeret Hall, Simon Caton, and Christof Weinhardt. 2013. Well-Being's Predictive Value: A Gamified Approach to Managing Smart Communities. In OCSC 2013: International Conference on Online
Communities and Social Computing, 13-22. https://doi.org/10.1007/978-3-642-39371-6_2

31. Juho Hamari and Jonna Koivisto. 2015. "Working out for likes": An empirical study on social influence in exercise gamification. Computers in Human Behavior 50: 333-347. https://doi.org/10.1016/j.chb.2015.04.018

32. Juho Hamari, Jonna Koivisto, and Tuomas Pakkanen. 2014. Do Persuasive Technologies Persuade? - A Review of Empirical Studies. In PERSUASIVE 2014: International Conference on Persuasive Technology, 118-136. https://doi.org/10.1007/978-3-319-07127-5_11

33. Juho Hamari, Jonna Koivisto, and Harri Sarsa. 2014. Does gamification work? - A literature review of empirical studies on gamification. In Proceedings of the Annual Hawaii International Conference on System Sciences, 3025-3034. https://doi.org/10.1109/HICSS.2014.377

34. Harri Oinas-Kukkonen and Marja Harjumaa. 2009. Persuasive Systems Design: Key Issues, Process Model, and System Features. Communications of the Association for Information Systems 24, 1. Retrieved September 8, 2017 from http://aisel.aisnet.org/cais/vol24/iss 1/28

35. Jacob B Hirsh, Sonia K Kang, and Galen V Bodenhausen. 2012. Personalized persuasion: tailoring persuasive appeals to recipients' personality traits. Psychological Science 23, 6: 578-81. https://doi.org/10.1177/0956797611436349

36. Astrid Jander, Rik Crutzen, Liesbeth Mercken, Math Candel, and Hein de Vries. 2016. Effects of a WebBased Computer-Tailored Game to Reduce Binge Drinking Among Dutch Adolescents: A Cluster Randomized Controlled Trial. Journal of Medical Internet Research 18, 2: e29. https://doi.org/10.2196/jmir.4708

37. Astrid Jander, Rik Crutzen, Liesbeth Mercken, and Hein De Vries. 2014. A Web-based computer-tailored game to reduce binge drinking among 16 to 18 year old Dutch adolescents: development and study protocol. BMC Public Health 14: 1054. https://doi.org/10.1186/1471-2458-14-1054

38. J B Jemmott, $3^{\text {rd }}$, L S Jemmott, and G T Fong. 1992. Reductions in HIV risk-associated sexual behaviors among black male adolescents: effects of an AIDS prevention intervention. American Journal of Public Health 82, 3: 372-377. https://doi.org/10.2105/AJPH.82.3.372

39. Yuan Jia, Bin Xu, Yamini Karanam, and Stephen Voida. 2016. Personality-targeted Gamification: A Survey Study on Personality Traits and Motivational Affordances. In Proceedings of the $34^{\text {th }}$ Annual ACM Conference on Human Factors in Computing Systems CHI'16. https://doi.org/10.1145/2858036.2858515 
40. Daniel Johnson, Sebastian Deterding, Kerri-Ann Kuhn, Aleksandra Staneva, Stoyan Stoyanov, and Leanne Hides. 2016. Gamification for health and wellbeing: A systematic review of the literature. Internet Interventions 6: 89-106. https://doi.org/10.1016/j.invent.2016.10.002

41. Henry F. Kaiser. 1970. A second generation little jiffy. Psychometrika 35, 4: 401-415. https://doi.org/10.1007/BF02291817

42. Maurits Kaptein, Boris De Ruyter, Panos Markopoulos, and Emile Aarts. 2012. Adaptive Persuasive Systems: A Study of Tailored Persuasive Text Messages to Reduce Snacking. ACM Transactions on Interactive Intelligent Systems 2, 2: 125. https://doi.org/10.1145/2209310.2209313

43. Rilla Khaled, Ronald Fischer, James Noble, and Robert Biddle. 2008. A qualitative study of culture and persuasion in a smoking cessation game. In Proceedings of the Third International Conference on Persuasive Technology for Human Well-Being, 224236. https://doi.org/10.1007/978-3-540-68504-3-20

44. Emil Kupek. 2006. Beyond logistic regression: structural equations modelling for binary variables and its application to investigating unobserved confounders. BMC Medical Research Methodology 6, 1: 13. https://doi.org/10.1186/1471-2288-6-13

45. Itaru Kuramoto, Takuya Ishibashi, Keiko Yamamoto, and Yoshihiro Tsujino. 2013. Stand Up, Heroes! : Gamification for Standing People on Crowded Public Transportation. In DUXU 2013: International Conference of Design, User Experience, and Usability, 538-547. https://doi.org/10.1007/978-3-642-39241-2_59

46. Tuomas Lehto and Harri Oinas-Kukkonen. 2011. Persuasive features in web-based alcohol and smoking interventions: a systematic review of the literature. Journal of Medical Internet Research 13, 3: e46. https://doi.org/10.2196/jmir.1559

47. Corrie Lelie. 2005. The value of storyboards in the product design process. Personal and Ubiquitous Computing 10, 2-3: 159-162.

https://doi.org/10.1007/s00779-005-0026-7

48. Edwin A. Locke and Gary P. Latham. 1994. Goalsetting Theory. In Organizational Behavior 1: Essential Theories of Motivation and Leadership, John B. Miner (ed.). Routledge, New York, NY, 159-183.

49. Edwin A. Locke and Gary P. Latham (eds.). 2013. New developments in goal setting and task performance. Routledge, New York, NY.

50. Geke D. S. Ludden, Saskia M. Kelders, and Bas H. J. Snippert. 2014. "This Is Your Life!”: The Design of a Positive Psychology Intervention Using Metaphor to Motivate. In PERSUASIVE 2014: International Conference on Persuasive Technology, 179-190. https://doi.org/10.1007/978-3-319-07127-5_16
51. Winter Mason and Siddharth Suri. 2012. Conducting behavioral research on Amazon's Mechanical Turk. Behavior Research Methods 44, 1: 1-23. https://doi.org/10.3758/s13428-011-0124-6

52. Elke Mattheiss, Marc Busch, Rita Orji, Gustavo F. Tondello, Andrzej Marczewski, Wolfgang Hochleitner, Michael Lankes, and Manfred Tscheligi. 2017. UMAP 2017 Fifty Shades of Personalization - Workshop on Personalization in Serious and Persuasive Games and Gameful Interactions : Organizers ' Welcome. In Proceedings of UMAP'17 Adjunct, 395-397.

53. Jo Mitchell, Kellie Vella, Daniel Johnson, Nicole Peever, Vanessa Wan Sze Cheng, Tracey Davenport, Jane Burns, Ian Hickie, Anne Kyle, Brent Hedley, and Brett Johnson. 2017. MindMax: Using Videogames and Sport to Engage Young Men and Improve Wellbeing. In $2^{\text {nd }}$ Symposium Computing and Mental Health. Retrieved from http://mentalhealth.media.mit.edu/wp-content/ uploads/sites/46/2017/05/CMH_2017_paper_16.pdf

54. Baptiste Monterrat, Élise Lavoué, and Sébastien George. 2015. Toward an Adaptive Gamification System for Learning Environments. In International Conference on Computer Supported Education, 115129. https://doi.org/10.1007/978-3-319-25768-6_8

55. Vandana Naik and Venkatesh Kamat. 2015. Adaptive and Gamified Learning Environment (AGLE). In 2015 IEEE Seventh International Conference on Technology for Education (T4E), 7-14. https://doi.org/10.1109/T4E.2015.23

56. Harri Oinas-Kukkonen and Marja Harjumaa. 2009. Persuasive Systems Design: Key Issues, Process Model, and System Features. Communications of the Association for Information Systems 24, 1: 28. Retrieved from http://aisel.aisnet.org/cais/vol24/iss1/28

57. Rita Orji. 2014. Design for Behaviour Change: $A$ Model-driven Approach for Tailoring Persuasive Technologies. University of Saskatchewan. Retrieved from http://hdl.handle.net/10388/ETD-2014-06-1555

58. Rita Orji, Regan L. Mandryk, and Julita Vassileva. 2017. Improving the Efficacy of Games for Change Using Personalization Models. ACM Transactions on Computer-Human Interaction 24, 5. https://doi.org/10.1145/3119929

59. Rita Orji, Regan L. Mandryk, Julita Vassileva, and Kathrin M. Gerling. 2013. Tailoring persuasive health games to gamer type. In Proceedings of the SIGCHI Conference on Human Factors in Computing Systems CHI '13, 2467-2476. https://doi.org/10.1145/2470654.2481341

60. Rita Orji and Karyn Moffatt. 2016. Persuasive technology for health and wellness: State-of-the-art and emerging trends. Health Informatics Journal. https://doi.org/10.1177/1460458216650979 
61. Rita Orji, Lennart E. Nacke, and Chrysanne DiMarco. 2017. Towards Personality-driven Persuasive Health Games and Gamified Systems. In Proceedings of the SIGCHI Conference on Human Factors in Computing Systems, 1015-1027. https://doi.org/10.1145/3025453.3025577

62. Rita Orji, Julita Vassileva, and Regan L. Mandryk. 2014. Modeling the efficacy of persuasive strategies for different gamer types in serious games for health. User Modeling and User-Adapted Interaction 24, 5: 453-498. https://doi.org/10.1007/s11257-014-9149-8

63. Ranilson Paiva, Ig Ibert Bittencourt, Thyago Tenório, Patricia Jaques, and Seiji Isotani. 2016. What do students do on-line? Modeling students' interactions to improve their learning experience. Computers in Human Behavior 64: 769-781. https://doi.org/10.1016/j.chb.2016.07.048

64. Amon Rapp. 2017. From Games to Gamification: A Classification of Rewards in World of Warcraft for the Design of Gamified Systems. Simulation \& Gaming 48, 3: 381-401. https://doi.org/10.1177/1046878117697147

65. Ganit Richter, Daphne R. Raban, and Sheizaf Rafaeli. 2015. Studying Gamification: The Effect of Rewards and Incentives on Motivation. In Gamification in Education and Business, Torsten Reiners and Lincoln C. Wood (eds.). Springer, Cham, 21-46. https://doi.org/10.1007/978-3-319-10208-5_2

66. Christian M. Ringle, S. Wende, and J. Becker. SmartPLS: Next Generation Path Modeling. Retrieved September 9, 2017 from https://www.smartpls.com/

67. Silvia Riva, Anne-Linda Camerini, Ahmed Allam, and Peter J Schulz. 2014. Interactive sections of an Internet-based intervention increase empowerment of chronic back pain patients: randomized controlled trial. Journal of Medical Internet Research 16, 8: e180. https://doi.org/10.2196/jmir.3474

68. Richard M. Ryan and Edward L. Deci. 2000. Selfdetermination theory and the facilitation of intrinsic motivation, social development, and well-being. The American Psychologist 55, 1: 68-78. https://doi.org/10.1037/0003-066X.55.1.68

69. Steven P Schinke, Traci M Schwinn, and Alfred J Ozanian. 2005. Alcohol abuse prevention among highrisk youth: computer-based intervention. Journal of Prevention \& Intervention in the Community 29, 1-2: 117-30. https://doi.org/10.1300/J005v29n01_08

70. Katie Seaborn and Deborah I. Fels. 2014. Gamification in theory and action: A survey. International Journal of Human-Computer Studies 74: 14-31. https://doi.org/10.1016/j.ijhcs.2014.09.006

71. B. F Skinner. 1953. Science And Human Behavior. Simon and Schuster. https://doi.org/10.1037/h0052427
72. Frank Spillers and Stavros Asimakopoulos. 2014. Does Social User Experience Improve Motivation for Runners? In DUXU 2014: International Conference of Design, User Experience, and Usability, 358-369. https://doi.org/10.1007/978-3-319-07638-6_35

73. S. Shyam Sundar and Sampada S. Marathe. 2010. Personalization versus Customization: The Importance of Agency, Privacy, and Power Usage. Human Communication Research 36, 3: 298-322. https://doi.org/10.1111/j.1468-2958.2010.01377.x

74. Ana Carolina Tomé Klock, Lucas Felipe da Cunha, Mayco Farias de Carvalho, Brayan Eduardo Rosa, Andressa Jaqueline Anton, and Isabela Gasparini. 2015. Gamification in e-Learning Systems: A Conceptual Model to Engage Students and Its Application in an Adaptive e-Learning System. In International Conference on Learning and Collaboration Technologies, 595-607. https://doi.org/10.1007/978-3-319-20609-7_56

75. Gustavo F. Tondello, Alberto Mora, and Lennart E. Nacke. 2017. Elements of Gameful Design Emerging from User Preferences. In Proceedings of the 2017 Annual Symposium on Computer-Human Interaction in Play - CHI PLAY'17. https://doi.org/10.1145/3116595.3116627

76. Gustavo F. Tondello, Rita Orji, and Lennart E. Nacke. 2017. Recommender Systems for Personalized Gamification. In Proceedings of UMAP'17 Adjunct. https://doi.org/10.1145/3099023.3099114

77. Gustavo F. Tondello, Rina R. Wehbe, Lisa Diamond, Marc Busch, Andrzej Marczewski, and Lennart E. Nacke. 2016. The Gamification User Types Hexad Scale. In Proceedings of the 2016 Annual Symposium on Computer-Human Interaction in Play - CHI PLAY '16. https://doi.org/10.1145/2967934.2968082

78. Khai N Truong, Gillian R Hayes, and Gregory D Abowd. 2006. Storyboarding: An Empirical Determination of Best Practices and Effective Guidelines. In Proceedings of the 6th conference on Designing Interactive systems (DIS '06), 12-21. https://doi.org/10.1145/1142405.1142410

79. Julita Vassileva. 2012. Motivating participation in social computing applications: a user modeling perspective. User Modeling and User-Adapted Interaction 22, 1-2: 177-201. https://doi.org/10.1007/s11257-011-9109-5

80. Oren Zuckerman and Ayelet Gal-Oz. 2014. Deconstructing gamification: evaluating the effectiveness of continuous measurement, virtual rewards, and social comparison for promoting physical activity. Personal and Ubiquitous Computing 18, 7: 1705-1719. https://doi.org/10.1007/s00779-014-0783-2 